Supporting information

\title{
Trigonal Prismatic Co(II) Single-Ion Magnets: Manipulating the Magnetic Relaxation Through Symmetry Control
}

Binling Yao, ${ }^{a}$ Mukesh Kumar Singh, ${ }^{\mathrm{b}}$ Yi-Fei Deng,,${ }^{\mathrm{a}, *}$ Yi-Nuo Wang, ${ }^{\mathrm{a}}$ Kim R Dunbar ${ }^{\mathrm{b}}$ and Yuan-Zhu Zhang,*

a Department of Chemistry, Southern University of Science and Technology (SUSTech), Shenzhen, 518055, China.

${ }^{\mathrm{b}}$ Department of Chemistry, Texas A \& M University, College Station, TX 77842, USA. 


\section{Experimental Section}

Materials and Physical Measurements. 6, 6', 6"-(methoxymethanetriyl)tris(2-(1H-pyrazol-1yl)pyridine) (tppm $\left.{ }^{*}\right)$ and tris(2, 2'-bipyrid-6-yl)methanol (hpy) were synthesized according to the literatures. ${ }^{1}$ All other chemicals and reagents were commercial available and used without further purification. FT-IR spectra were recorded in the range $600-4000 \mathrm{~cm}^{-1}$ on a Bruker tensor II spectrophotometer. Elemental analyses $(\mathrm{C}, \mathrm{H}, \mathrm{N})$ were measured using an Elemental vario EL analyser. Powder X-ray diffraction (PXRD) measurements were recorded on a Rigaku Smartlab Xray diffractometer (Figures S1). A PXRD pattern for 2 could not be obtained due to the loss of $\mathrm{CH}_{2} \mathrm{Cl}_{2}$ interstitial solvent molecules. Magnetic susceptibility data were collected with a SQUID MPMS3 magnetometer. Diamagnetic corrections were calculated from Pascal constants and applied to all the constituent atoms and sample holder. ${ }^{2}$

Synthesis of $\left[\mathbf{C o}\left(t_{p p m} *\right)\right]\left[\mathbf{B P h}_{4}\right]_{2}$ (1). A methanolic solution (5 ml) containing $\mathrm{Co}\left(\mathrm{ClO}_{4}\right)_{2} \cdot 6 \mathrm{H}_{2} \mathrm{O}$ $(0.1 \mathrm{mmol}, 0.037 \mathrm{~g})$ and tppm* $(0.1 \mathrm{mmol}, 0.047 \mathrm{~g})$ was allowed to stir for $30 \mathrm{~min}$ and filtered. The remaining solid was dissolved in $20 \mathrm{ml} \mathrm{MeCN} / \mathrm{MeOH}$ (v/v, 1:9), to which excessive $\mathrm{NaBPh}_{4}(0.3$ mmol, $0.097 \mathrm{~g}$ ) was added. The mixture was continuously stirred for $30 \mathrm{~min}$ and yellow microcrystals were precipitated, which were filtered and washed by $\mathrm{MeOH}$ for several times. (0.066g, yield: $\sim 56 \%)$. Suitable single crystals for X-ray diffraction were obtained by recrystallization of a concentrated solution of 1 in $\mathrm{MeCN}$. Anal. calcd (\%): $\mathrm{C}_{74} \mathrm{H}_{61} \mathrm{~B}_{2} \mathrm{CoN} \mathrm{N}_{9} \mathrm{O}$ : C, 75.78; H, 5.24; N, 10.75. Found (\%): C, 75.90; H, 5.65; N, 10.79. IR (KBr, $\left.v / \mathrm{cm}^{-1}\right): 3120(\mathrm{~m})$, 1608(s), 1577(vs), 1525(vs), 1477(s), 1464(s), 1402(vs), 1350(m), 1066(vs), 964(m), 804(m), 729(s), 702(vs), 671(m), 644(m), 607(w).

Synthesis of [Co(hpy)] [BPh $\left.]_{4}\right]_{2} \cdot \mathbf{C C H}_{\mathbf{2}} \mathbf{C l}_{\mathbf{2}}$ (2). Complex $\mathbf{2}$ was obtained in a similar way to complex 1 by using the hpy ligand and recrystallization in $\mathrm{CH}_{2} \mathrm{Cl}_{2}$. (0.042g, yield: $\left.\sim 29 \%\right)$. Anal. calcd (\%): $\mathrm{C}_{82} \mathrm{H}_{68} \mathrm{~B}_{2} \mathrm{Cl}_{6} \mathrm{CoN}_{6} \mathrm{O}: \mathrm{C}, 68.08 ; \mathrm{H}, 4.74 ; \mathrm{N}, 5.81 \%$. Found (\%): C, 68.48; H, 4.88; N, 6.05\%. IR (KBr, $\left.v / \mathrm{cm}^{-1}\right): 3448(\mathrm{w}), 3054(\mathrm{~m}), 1599(\mathrm{~s}), 1580(\mathrm{~s}), 1562(\mathrm{~s}), 1479(\mathrm{~s}), 1454(\mathrm{vs}), 1423(\mathrm{~s}), 1261(\mathrm{vs})$, 1018(vs), 769(s), 733(vs), 704(vs), 675(vs), 611(vs).

Crystallography. The diffraction data for $\mathbf{1}$ and $\mathbf{2}$ were collected at 100(2) K on a Bruker D8 VENTURE diffractometer with graphite monochromated Mo K $\alpha$ radiation $(\lambda=0.71073 \AA)$. The structures were solved by the direct method of SHELXT structure solution program and refined by full-matrix least squares (SHELXL) on $F^{2}$ and empirical absorption corrections (SADABS) were applied. ${ }^{3}$ Anisotropic thermal parameters were used for the non-hydrogen atoms. Hydrogen atoms were added geometrically and refined using a riding model. Weighted $\mathrm{R}$ factors $(w R)$ and all the goodness-of-fit $(S)$ values are based on $F^{2}$; conventional $\mathrm{R}$ factors $(R)$ are based on $\mathrm{F}$, with $\mathrm{F}$ set to zero for negative $F^{2}$. Data collection and structural refinement parameters are given in Table S1. Selected bond length and angles are given in Table S2 and S3. CCDC-1964736 (1) and 1964737 (2) contain the crystallographic data that can be obtained via www.ccdc.cam.ac.uk/conts/retrieving.html (or from the Cambridge Crystallographic Data Centre, 12, Union Road, Cambridge CB21EZ, UK; fax: (+44) 1223-336-033; or deposit@ccdc.cam.ac.uk).

Computational Details. Zero-field splitting parameters (D, E, and g-tensor) calculations were performed using two different software packages, ORCA (ORCA 4.1.0) ${ }^{4}$ and MOLCAS (MOLCAS $8.1),{ }^{5}$ on the XRD structures for 1-2. Ab initio SA-CASSCF/NEVPT2 calculations were carried out using the ORCA suite. ${ }^{6}$ The zeroth - order regular approximation (ZORA) method was used which 
considers the scalar relativistic effects. ${ }^{7}$ Additionally, ZORA contracted versions of basis sets ZORA - def2 - TZVPP were used for Co, and the ZORA - def2 - TZVP basis set was used for the other atoms. ${ }^{8}$ We considered five d-orbitals with seven electrons (CAS $(7,5)$ setup) in the active space for the state-average complete active space self-consistent field (SA-CASSCF) calculations; ten quartet and forty doublet roots were used. ${ }^{6}$ Dynamic correlations were included by performing the second-order $\mathrm{N}$ - electron valence perturbation theory (NEVPT2) calculation ${ }^{9}$ which is known to give accurate estimations of the ZFS parameters. ${ }^{10}$ The analysis was limited to the NEVPT2 results.

The $a b$ initio CASSCF + RASSI-SO/SINGLE_ANISO calculations were also performed using the MOLCAS 8 code to estimate anisotropy parameters. ${ }^{5,11}$ Here the [ANO-RCC-VTZP] basis set was employed for the Co atom, the [ANO-RCC-VDZP] basis set for the $\mathrm{N}$ atoms, and the [ANORCC-VDZ] basis set for the $\mathrm{C}$ and $\mathrm{H}$ atoms. CASSCF active space calculations are composed of seven electrons with five active orbitals $\operatorname{CAS}(7,5)$. Ten quartets and forty doublets were computed using the configuration interaction $(\mathrm{CI})$ procedure. Then, the RASSI-SO module was used to compute the spin-orbit coupled states and the SINGLE_ANISO code was performed to extract the corresponding anisotropy parameters. ${ }^{12}$

Table S1. Crystallographic data for 1 and 2.

\begin{tabular}{|c|c|c|}
\hline & 1 & 2 \\
\hline Formula & $\mathrm{C}_{74} \mathrm{H}_{61} \mathrm{~B}_{2} \mathrm{CoN}_{9} \mathrm{O}$ & $\mathrm{C}_{82} \mathrm{H}_{68} \mathrm{~B}_{2} \mathrm{Cl}_{6} \mathrm{CoN}_{6} \mathrm{O}$ \\
\hline Molecular weight $/ \mathrm{g} \mathrm{mol}^{-1}$ & 1172.86 & 1446.67 \\
\hline Crystal system & Orthorhombic & Triclinic \\
\hline Space group & $P 2_{1} 2_{1} 2_{1}$ & $P-1$ \\
\hline $\mathrm{a}, \AA$ & $11.7586(5)$ & $13.7814(15)$ \\
\hline $\mathrm{b}, \AA$ & $20.7508(11)$ & $13.9635(18)$ \\
\hline $\mathrm{c}, \AA$ & $24.5008(12)$ & 21.691(3) \\
\hline$\alpha, \operatorname{deg}$ & 90 & $100.740(5)$ \\
\hline$\beta, \operatorname{deg}$ & 90 & $91.677(4)$ \\
\hline$\gamma, \operatorname{deg}$ & 90 & $119.001(3)$ \\
\hline $\mathrm{V}, \AA^{3}$ & $5978.2(5)$ & $3551.9(8)$ \\
\hline Z & 4 & 2 \\
\hline$D_{\text {cal }} / \mathrm{g} \mathrm{cm}^{-3}$ & 1.303 & 1.353 \\
\hline temperature, $\mathrm{K}$ & $100(2)$ & $100(2)$ \\
\hline$\theta$ range $/{ }^{\circ}$ & $2.40-24.74^{\circ}$ & $2.27-24.79^{\circ}$ \\
\hline completeness & $99.8 \%$ & $99.1 \%$ \\
\hline residual map, e $\AA^{-3}$ & $2.133 /-0.459$ & $0.756 /-0.598$ \\
\hline Goodness-of-fit on $\mathrm{F}^{2}$ & 1.099 & 0.998 \\
\hline Final indices $[\mathrm{I}>2 \sigma(\mathrm{I})]^{\mathrm{a}, \mathrm{b}}$ & $R_{1}=0.0710, w R_{2}=0.1885$ & $R_{1}=0.0617, w R_{2}=0.1324$ \\
\hline$R$ indices (all data) & $R_{1}=0.0804, w R_{2}=0.1976$ & $R_{1}=0.0997, w R_{2}=0.1488$ \\
\hline
\end{tabular}


Table S2. Selected bond lengths $[\AA]$ and angles [deg] for $\mathbf{1}$.

\begin{tabular}{llll}
\hline $\operatorname{Co}(1)-\mathrm{N}(1)$ & $2.112(6)$ & $\mathrm{Co}(1)-\mathrm{N}(3)$ & $2.134(5)$ \\
$\operatorname{Co}(1)-\mathrm{N}(4)$ & $2.134(6)$ & $\mathrm{Co}(1)-\mathrm{N}(6)$ & $2.117(6)$ \\
$\operatorname{Co}(1)-\mathrm{N}(7)$ & $2.111(6)$ & $\mathrm{Co}(1)-\mathrm{N}(9)$ & $2.173(6)$ \\
$\mathrm{N}(7)-\mathrm{Co}(1)-\mathrm{N}(1)$ & $94.3(2)$ & $\mathrm{N}(6)-\mathrm{Co}(1)-\mathrm{N}(3)$ & $81.0(2)$ \\
$\mathrm{N}(7)-\mathrm{Co}(1)-\mathrm{N}(4)$ & $93.2(2)$ & $\mathrm{N}(7)-\mathrm{Co}(1)-\mathrm{N}(9)$ & $75.0(2)$ \\
$\mathrm{N}(1)-\mathrm{Co}(1)-\mathrm{N}(4)$ & $86.7(2)$ & $\mathrm{N}(6)-\mathrm{Co}(1)-\mathrm{N}(9)$ & $82.6(2)$ \\
$\mathrm{N}(6)-\mathrm{Co}(1)-\mathrm{N}(4)$ & $75.7(2)$ & $\mathrm{N}(3)-\mathrm{Co}(1)-\mathrm{N}(9)$ & $81.2(2)$ \\
$\mathrm{N}(1)-\mathrm{Co}(1)-\mathrm{N}(3)$ & $75.1(2)$ & & \\
\hline
\end{tabular}

Table S3. Selected bond lengths $[\AA]$ and angles [deg] for $\mathbf{2}$.

\begin{tabular}{llll}
\hline $\mathrm{Co}(1)-\mathrm{N}(1)$ & $2.134(3)$ & $\mathrm{Co}(1)-\mathrm{N}(2)$ & $2.108(3)$ \\
$\mathrm{Co}(1)-\mathrm{N}(3)$ & $2.171(3)$ & $\mathrm{Co}(1)-\mathrm{N}(4)$ & $2.104(3)$ \\
$\mathrm{Co}(1)-\mathrm{N}(5)$ & $2.177(3)$ & $\mathrm{Co}(1)-\mathrm{N}(6)$ & $2.105(3)$ \\
$\mathrm{N}(4)-\mathrm{Co}(1)-\mathrm{N}(6)$ & $83.62(11)$ & $\mathrm{N}(1)-\mathrm{Co}(1)-\mathrm{N}(3)$ & $91.15(12)$ \\
$\mathrm{N}(4)-\mathrm{Co}(1)-\mathrm{N}(2)$ & $81.71(12)$ & $\mathrm{N}(6)-\mathrm{Co}(1)-\mathrm{N}(5)$ & $75.79(11)$ \\
$\mathrm{N}(6)-\mathrm{Co}(1)-\mathrm{N}(2)$ & $83.51(12)$ & $\mathrm{N}(1)-\mathrm{Co}(1)-\mathrm{N}(5)$ & $94.57(11)$ \\
$\mathrm{N}(2)-\mathrm{Co}(1)-\mathrm{N}(1)$ & $75.58(11)$ & $\mathrm{N}(3)-\mathrm{Co}(1)-\mathrm{N}(5)$ & $91.81(11)$ \\
$\mathrm{N}(4)-\mathrm{Co}(1)-\mathrm{N}(3)$ & $75.80(12)$ & & \\
\hline
\end{tabular}

Table S4. Parameters fitted by a generalized Debye model at $8 \mathrm{~K}$ and different de fields for $\mathbf{1}$.

\begin{tabular}{ccc}
\hline$H / \mathrm{kOe}$ & $\tau / \mathrm{s}$ & $\alpha$ \\
\hline 0 & $4.23 \times 10^{-3}$ & 0.19 \\
0.2 & $4.30 \times 10^{-3}$ & 0.22 \\
0.4 & $5.22 \times 10^{-3}$ & 0.18 \\
0.6 & $6.23 \times 10^{-3}$ & 0.13 \\
0.8 & $6.52 \times 10^{-3}$ & 0.12 \\
1.0 & $6.58 \times 10^{-3}$ & 0.11 \\
1.5 & $6.63 \times 10^{-3}$ & 0.11 \\
2.0 & $6.45 \times 10^{-3}$ & 0.09 \\
2.5 & $6.42 \times 10^{-3}$ & 0.08 \\
3.0 & $6.34 \times 10^{-3}$ & 0.09 \\
4.0 & $5.99 \times 10^{-3}$ & 0.06 \\
5.0 & $5.75 \times 10^{-3}$ & 0.05 \\
\hline
\end{tabular}

Table S5. Parameters fitted by a generalized Debye model at zero dc field for $\mathbf{1}$.

\begin{tabular}{ccc}
\hline$T / \mathrm{K}$ & $\tau / \mathrm{s}$ & $\alpha$ \\
\hline 6.0 & $2.26 \times 10^{-2}$ & 0.24 \\
6.5 & $1.42 \times 10^{-2}$ & 0.22 \\
7.0 & $9.45 \times 10^{-3}$ & 0.19 \\
7.5 & $6.32 \times 10^{-3}$ & 0.18 \\
8.0 & $4.33 \times 10^{-3}$ & 0.17 \\
8.5 & $3.11 \times 10^{-3}$ & 0.17 \\
\hline
\end{tabular}




\begin{tabular}{ccc}
9.0 & $2.33 \times 10^{-3}$ & 0.15 \\
9.5 & $1.76 \times 10^{-3}$ & 0.14 \\
10.0 & $1.36 \times 10^{-3}$ & 0.12 \\
10.5 & $1.08 \times 10^{-3}$ & 0.11 \\
11.0 & $8.44 \times 10^{-4}$ & 0.10 \\
11.5 & $6.76 \times 10^{-4}$ & 0.09 \\
12.0 & $5.37 \times 10^{-4}$ & 0.08 \\
12.5 & $4.30 \times 10^{-4}$ & 0.08 \\
13.0 & $3.36 \times 10^{-4}$ & 0.08 \\
13.5 & $2.63 \times 10^{-4}$ & 0.08 \\
14.0 & $2.01 \times 10^{-4}$ & 0.08 \\
14.5 & $1.53 \times 10^{-4}$ & 0.09 \\
15.0 & $1.06 \times 10^{-4}$ & 0.10 \\
15.5 & $6.19 \times 10^{-5}$ & 0.14 \\
16.0 & $4.26 \times 10^{-5}$ & 0.13 \\
\hline
\end{tabular}

Table S6. Parameters fitted by a generalized Debye model at 1000 Oe dc field for $\mathbf{1}$.

\begin{tabular}{ccc}
\hline$T / \mathrm{K}$ & $\tau / \mathrm{s}$ & $\alpha$ \\
\hline 5.0 & $1.81 \times 10^{-1}$ & 0.23 \\
5.5 & $8.08 \times 10^{-2}$ & 0.20 \\
6.0 & $4.26 \times 10^{-2}$ & 0.17 \\
6.5 & $2.45 \times 10^{-2}$ & 0.15 \\
7.0 & $1.50 \times 10^{-2}$ & 0.13 \\
7.5 & $9.68 \times 10^{-3}$ & 0.12 \\
8.0 & $6.54 \times 10^{-3}$ & 0.11 \\
8.5 & $4.55 \times 10^{-3}$ & 0.10 \\
9.0 & $3.25 \times 10^{-3}$ & 0.10 \\
9.5 & $2.39 \times 10^{-3}$ & 0.09 \\
10.0 & $1.78 \times 10^{-3}$ & 0.09 \\
10.5 & $1.35 \times 10^{-3}$ & 0.08 \\
11.0 & $1.05 \times 10^{-3}$ & 0.08 \\
11.5 & $7.88 \times 10^{-4}$ & 0.08 \\
12.0 & $6.04 \times 10^{-4}$ & 0.08 \\
12.5 & $4.65 \times 10^{-4}$ & 0.08 \\
13.0 & $3.60 \times 10^{-4}$ & 0.07 \\
13.5 & $2.79 \times 10^{-4}$ & 0.07 \\
14.0 & $2.12 \times 10^{-4}$ & 0.07 \\
14.5 & $1.65 \times 10^{-4}$ & 0.04 \\
15.0 & $1.29 \times 10^{-4}$ & 0.01 \\
15.5 & $1.00 \times 10^{-4}$ & 0.01 \\
16.0 & $7.71 \times 10^{-5}$ & 0.01 \\
\hline
\end{tabular}


Table S7. Parameters fitted by a generalized Debye model at $3 \mathrm{~K}$ and different dc fields for $\mathbf{2}$.

\begin{tabular}{ccc}
\hline$H / \mathrm{kOe}$ & $\tau / \mathrm{s}$ & $\alpha$ \\
\hline 0 & $1.17 \times 10^{-3}$ & 0.29 \\
0.5 & $2.62 \times 10^{-1}$ & 0.34 \\
1.0 & $3.81 \times 10^{-1}$ & 0.29 \\
1.5 & $2.60 \times 10^{-1}$ & 0.21 \\
2.0 & $1.93 \times 10^{-1}$ & 0.16 \\
2.5 & $1.59 \times 10^{-1}$ & 0.15 \\
3.0 & $1.16 \times 10^{-1}$ & 0.12 \\
4.0 & $6.81 \times 10^{-2}$ & 0.11 \\
5.0 & $3.52 \times 10^{-2}$ & 0.11 \\
\hline
\end{tabular}

Table S8. Parameters fitted by a generalized Debye model at zero dc field for $\mathbf{2}$.

\begin{tabular}{|c|c|c|}
\hline$T / \mathrm{K}$ & $\tau / \mathrm{s}$ & $\alpha$ \\
\hline 2.00 & $1.21 \times 10^{-3}$ & 0.26 \\
\hline 2.25 & $1.19 \times 10^{-3}$ & 0.26 \\
\hline 2.50 & $1.17 \times 10^{-3}$ & 0.26 \\
\hline 2.75 & $1.15 \times 10^{-3}$ & 0.27 \\
\hline 3.00 & $1.12 \times 10^{-3}$ & 0.27 \\
\hline 3.25 & $1.11 \times 10^{-3}$ & 0.27 \\
\hline 3.50 & $1.10 \times 10^{-3}$ & 0.27 \\
\hline 3.75 & $1.08 \times 10^{-3}$ & 0.27 \\
\hline 4.00 & $1.05 \times 10^{-3}$ & 0.27 \\
\hline 4.25 & $1.03 \times 10^{-3}$ & 0.27 \\
\hline 4.50 & $1.00 \times 10^{-3}$ & 0.27 \\
\hline 4.75 & $9.86 \times 10^{-4}$ & 0.26 \\
\hline 5.00 & $9.38 \times 10^{-4}$ & 0.26 \\
\hline 5.25 & $9.30 \times 10^{-4}$ & 0.24 \\
\hline 5.50 & $8.63 \times 10^{-4}$ & 0.24 \\
\hline 5.75 & $8.38 \times 10^{-4}$ & 0.24 \\
\hline 6.00 & $7.81 \times 10^{-4}$ & 0.23 \\
\hline 6.25 & $7.55 \times 10^{-4}$ & 0.22 \\
\hline 6.50 & $7.12 \times 10^{-4}$ & 0.21 \\
\hline 6.75 & $6.08 \times 10^{-4}$ & 0.22 \\
\hline 7.00 & $6.02 \times 10^{-4}$ & 0.19 \\
\hline 7.25 & $5.47 \times 10^{-4}$ & 0.19 \\
\hline 7.50 & $5.08 \times 10^{-4}$ & 0.17 \\
\hline 7.75 & $4.60 \times 10^{-4}$ & 0.17 \\
\hline 8.00 & $4.24 \times 10^{-4}$ & 0.16 \\
\hline 8.25 & $3.85 \times 10^{-4}$ & 0.15 \\
\hline 8.50 & $3.07 \times 10^{-4}$ & 0.17 \\
\hline
\end{tabular}




\begin{tabular}{ccc}
8.75 & $2.93 \times 10^{-4}$ & 0.15 \\
9.00 & $2.86 \times 10^{-4}$ & 0.13 \\
9.25 & $2.51 \times 10^{-4}$ & 0.12 \\
9.50 & $2.27 \times 10^{-4}$ & 0.13 \\
9.75 & $2.12 \times 10^{-4}$ & 0.10 \\
10.00 & $2.05 \times 10^{-4}$ & 0.08 \\
\hline
\end{tabular}

Table S9. Parameters fitted by a generalized Debye model at 1000 Oe dc field for 2.

\begin{tabular}{ccc}
\hline$T / \mathrm{K}$ & $\tau / \mathrm{s}$ & $\alpha$ \\
\hline 3.75 & $7.85 \times 10^{-2}$ & 0.17 \\
4.00 & $5.20 \times 10^{-2}$ & 0.14 \\
4.25 & $3.63 \times 10^{-2}$ & 0.13 \\
4.50 & $2.58 \times 10^{-2}$ & 0.11 \\
4.75 & $1.91 \times 10^{-2}$ & 0.11 \\
5.00 & $1.40 \times 10^{-2}$ & 0.10 \\
5.25 & $1.07 \times 10^{-2}$ & 0.10 \\
5.50 & $8.20 \times 10^{-3}$ & 0.10 \\
5.75 & $6.44 \times 10^{-3}$ & 0.10 \\
6.00 & $5.06 \times 10^{-3}$ & 0.09 \\
6.25 & $3.99 \times 10^{-3}$ & 0.09 \\
6.50 & $3.20 \times 10^{-3}$ & 0.10 \\
6.75 & $2.60 \times 10^{-3}$ & 0.10 \\
7.00 & $2.13 \times 10^{-3}$ & 0.10 \\
7.25 & $1.74 \times 10^{-3}$ & 0.10 \\
7.50 & $1.44 \times 10^{-3}$ & 0.10 \\
7.75 & $1.19 \times 10^{-3}$ & 0.10 \\
8.00 & $1.02 \times 10^{-3}$ & 0.10 \\
8.25 & $8.65 \times 10^{-4}$ & 0.10 \\
8.50 & $7.40 \times 10^{-4}$ & 0.08 \\
8.75 & $6.42 \times 10^{-4}$ & 0.07 \\
9.00 & $5.62 \times 10^{-4}$ & 0.07 \\
9.25 & $4.92 \times 10^{-4}$ & 0.05 \\
9.50 & $4.39 \times 10^{-4}$ & 0.05 \\
9.75 & $3.91 \times 10^{-4}$ & 0.03 \\
10.0 & $3.49 \times 10^{-4}$ & 0.02 \\
\hline & &
\end{tabular}

The equation employed for Cole-Cole data ${ }^{13}$ :

$$
\begin{aligned}
& \chi^{\prime}(\omega)=\chi_{S}+\frac{\left(\chi_{T}-\chi_{S}\right)\left[1+(\omega \tau)^{1-\alpha} \sin (\alpha \pi / 2)\right]}{1+2(\omega \tau)^{1-\alpha} \sin (\alpha \pi / 2)+(\omega \tau)^{2(1-\alpha)}} \\
& \chi^{\prime \prime}(\omega)=\chi_{S}+\frac{\left(\chi_{T}-\chi_{S}\right)(\omega \tau)^{1-\alpha} \cos (\alpha \pi / 2)}{1+2(\omega \tau)^{1-\alpha} \sin (\alpha \pi / 2)+(\omega \tau)^{2(1-\alpha)}}
\end{aligned}
$$


Table S10. Selected parameters for reported trigonal prismatic Co(II) SIMs.

\begin{tabular}{|c|c|c|c|c|}
\hline Compound & $D / \mathrm{cm}^{-1}$ & $E / \mathrm{cm}^{-1}$ & $U_{\text {eff }} / \mathrm{cm}^{-1}$ & ref \\
\hline$\left(\mathrm{HNEt}_{3}\right)^{+}\left(\mathrm{Co}^{\mathrm{II}} \mathrm{Co}^{\mathrm{III}}{ }_{3} \mathrm{~L}_{6}\right)^{-}$ & -115 & 2.8 & 76 & 14 \\
\hline$\left[\mathrm{Co}\left(\mathrm{P}(\mathrm{S})\left(\mathrm{N}_{3}\right)\right]\left[\left(\mathrm{NO}_{3}\right)_{2}\right]\right.$ & -72 & 7 & 23 & 15 \\
\hline$\left[\mathrm{Co}(\mathrm{PzOx})_{3}\left(\mathrm{BC}_{6} \mathrm{H}_{5}\right)\right] \mathrm{Cl} \cdot \mathrm{CHCl}_{3}$ & -82 & 0.246 & 152 & 16 \\
\hline$\left[\mathrm{H}\left(\mathrm{NEt}_{3}\right)\right]^{+}\left[\mathrm{Co}^{\mathrm{II}} \mathrm{Co}^{\mathrm{III}}{ }_{3}\left(\mathrm{~L}_{\mathrm{R}}^{1}\right)_{6}\right]^{-}$ & -25 & 0.05 & 27 & \multirow{7}{*}{17} \\
\hline$[\mathrm{H}(\mathrm{DBU})]^{+}\left[\mathrm{Co}^{\mathrm{II}} \mathrm{Co}_{3}^{\mathrm{III}}{ }_{3}\left(\mathrm{~L}_{\mathrm{R}}^{1}\right)_{6}\right]^{-}$ & -35 & 0.17 & 36 & \\
\hline$\left[\mathrm{H}\left(\mathrm{NEt}_{3}\right)\right]^{+}\left[\mathrm{Co}^{\mathrm{II}} \mathrm{Co}^{\mathrm{III}}{ }_{3}\left(\mathrm{~L}_{\mathrm{R}}^{2}\right)_{6}\right]^{-}$ & -22 & 0.03 & 30 & \\
\hline$[\mathrm{H}(\mathrm{DBU})]^{+}\left[\mathrm{Co}^{\mathrm{II}} \mathrm{Co}_{3}{ }_{3}\left(\mathrm{~L}_{\mathrm{R}}^{3}\right)_{6}\right]^{-}$ & $-b$ & - & 88 & \\
\hline$[\mathrm{H}(\mathrm{DIPEA})]^{+}\left[\mathrm{Co}^{\mathrm{II}} \mathrm{Co}_{3}^{\mathrm{III}}{ }_{3}\left(\mathrm{~L}_{\mathrm{R}}^{4}\right)_{6}\right]^{-}$ & - & - & 90 & \\
\hline$[\mathrm{H}(\mathrm{DBU})]^{+}\left[\mathrm{Co}^{\mathrm{II}} \mathrm{Co}_{3}{ }_{3}\left(\mathrm{~L}_{\mathrm{R}}^{4}\right)_{6}\right]^{-}$ & - & - & 95 & \\
\hline$\left(\mathrm{n}-\mathrm{Bu}_{4} \mathrm{~N}\right)^{+}\left[\mathrm{Co}^{\mathrm{II}} \mathrm{Co}_{3}{ }^{\mathrm{III}}\left(\mathrm{L}_{\mathrm{R}}^{4}\right)_{6}\right]^{-}$ & - & - & 102 & \\
\hline $\mathrm{Co}(\mathrm{SDZ})_{2}$ bpy & - & - & $45^{\mathrm{a}}$ & 18 \\
\hline$\left[\mathrm{Co}^{\mathrm{II}}(\mathrm{L})\right]$ & -31 & $4.0 \times 10^{-4}$ & $57^{\mathrm{a}}$ & 19 \\
\hline$\alpha-\mathrm{Co}$ & -111 & - & 192 & \multirow{2}{*}{20} \\
\hline$\beta-\mathrm{Co}$ & -74 & - & 131 & \\
\hline$\{\mathrm{Na}[\mathrm{LCo}]\}\left(\mathrm{BPh}_{4}\right)_{3}$ & -76 & $9 \times 10^{-4}$ & 52 & 21 \\
\hline$\left[\mathrm{Co}(\mathrm{AcPyOx})_{3} \mathrm{BC}_{6} \mathrm{H}_{5}\right] \mathrm{ClO}_{4}$ & -86 & - & 45 & 22 \\
\hline$[\mathrm{Co}(\mathrm{tppm})]\left[\mathrm{ClO}_{4}\right]_{2} \cdot 2 \mathrm{CH}_{3} \mathrm{CN} \cdot \mathrm{H}_{2} \mathrm{O}$ & -81 & 0.6 & 39 & $1 d$ \\
\hline 1 & -97 & $9.3 \times 10^{-3}$ & 192 & \multirow{2}{*}{ This work } \\
\hline 2 & -107 & 3.5 & 20 & \\
\hline
\end{tabular}

${ }^{\mathrm{a}}$ In an applied magnetic field; ${ }^{\mathrm{b}}$ Not reported.

Table S11. Ab initio NEVPT2 transition energies of the ligand field states (roots) for 1 along with its individual contributions to the $D$ and $E$ parameters.

$$
D=-97.3 \mathrm{~cm}^{-1} \quad E / D=0.002
$$

$g_{\mathrm{xx}}, g_{\mathrm{yy}}, g_{\mathrm{zz}}=2.003,2.009,3.097 \quad$ SOC constant zeta $=516 \mathrm{~cm}^{-1}$

\begin{tabular}{ccccc}
\hline NEVPT2 energy levels & Multiplicity & Root & Contribution to $D \mathrm{~cm}^{-1}$ & Contribution to $E \mathrm{~cm}^{-1}$ \\
\hline 0.0 & 4 & 0 & 0.0 & 0.0 \\
693.5 & 4 & 1 & -114.0 & 0.0 \\
9488.7 & 4 & 2 & 5.1 & -4.0 \\
9629.5 & 4 & 3 & 4.8 & 2.9 \\
10489.4 & 4 & 4 & 1.2 & 0.3 \\
10942.2 & 4 & 5 & 0.8 & 0.6 \\
12275.9 & 4 & 6 & 0.1 & 0.0 \\
15133.4 & 4 & 7 & 0.0 & 0.0 \\
15596.6 & 4 & 8 & 0.0 & 0.0 \\
18187.7 & 4 & 9 & 0.0 & 0.0 \\
18310.6 & 2 & 0 & -0.8 & 0.9 \\
18604.9 & 2 & 1 & -0.8 & -0.8 \\
19033.4 & 2 & 2 & 0.0 & 0.0 \\
19145.3 & 2 & 3 & 0.0 & 0.0 \\
22191.3 & 2 & 4 & 0.0 & 0.0
\end{tabular}




\begin{tabular}{|c|c|c|c|c|}
\hline 22978.4 & 2 & 5 & 0.0 & 0.0 \\
\hline 23061.0 & 2 & 6 & 7.3 & 0.0 \\
\hline 23382.2 & 2 & 7 & -0.4 & 0.1 \\
\hline 24298.4 & 2 & 8 & -1.8 & -1.5 \\
\hline 24575.1 & 2 & 9 & -1.5 & 1.3 \\
\hline 25262.7 & 2 & 10 & 1.4 & 0.0 \\
\hline 25658.0 & 2 & 11 & 0.0 & 0.0 \\
\hline 27801.4 & 2 & 12 & 0.0 & 0.0 \\
\hline 27879.7 & 2 & 13 & 0.0 & 0.0 \\
\hline 28409.9 & 2 & 14 & 0.0 & 0.0 \\
\hline 28858.2 & 2 & 15 & 0.0 & 0.0 \\
\hline 28875.1 & 2 & 16 & 0.0 & 0.0 \\
\hline 29465.1 & 2 & 17 & -0.3 & -0.2 \\
\hline 29695.4 & 2 & 18 & -0.1 & 0.1 \\
\hline 31094.4 & 2 & 19 & 0.0 & 0.0 \\
\hline 31158.0 & 2 & 20 & -0.1 & 0.1 \\
\hline 32039.5 & 2 & 21 & -0.9 & 0.7 \\
\hline 32412.0 & 2 & 22 & -0.9 & -0.6 \\
\hline 34931.9 & 2 & 23 & 0.0 & 0.0 \\
\hline 37199.3 & 2 & 24 & 0.0 & 0.0 \\
\hline 37321.4 & 2 & 25 & 0.0 & 0.0 \\
\hline 38236.5 & 2 & 26 & 0.1 & 0.0 \\
\hline 38691.6 & 2 & 27 & 0.0 & 0.0 \\
\hline 40866.9 & 2 & 28 & 0.4 & 0.0 \\
\hline 40917.6 & 2 & 29 & 0.0 & 0.0 \\
\hline 42350.0 & 2 & 30 & -0.1 & 0.1 \\
\hline 42705.6 & 2 & 31 & -0.1 & -0.1 \\
\hline 43590.2 & 2 & 32 & 0.0 & 0.0 \\
\hline 43753.0 & 2 & 33 & 0.0 & 0.0 \\
\hline 46334.3 & 2 & 34 & 0.0 & 0.0 \\
\hline 60710.9 & 2 & 35 & 0.0 & 0.0 \\
\hline 62821.7 & 2 & 36 & 0.0 & 0.0 \\
\hline 63650.5 & 2 & 37 & 0.0 & 0.0 \\
\hline 65402.6 & 2 & 38 & 0.0 & 0.0 \\
\hline 65659.9 & 2 & 39 & 0.1 & 0.0 \\
\hline
\end{tabular}

Table S12. Ab initio NEVPT2 transition energies of the ligand field states (roots) for $\mathbf{2}$ along with its individual contributions to the $D$ and $E$ parameters.

\begin{tabular}{ccccc}
\hline \multicolumn{2}{c}{$\begin{array}{c}g_{\mathrm{xx}}, g_{\mathrm{yy}}, g_{\mathrm{zz}}=1.896,1.934,3.216 \\
\text { NEVPT2 energy levels }\end{array}$} & Multiplicity & Root & SOC constant zeta $=515 \mathrm{~cm}^{-1}$ \\
\hline 0.0 & 4 & 0 & Contribution to $D \mathrm{~cm}^{-1}$ & Contribution to $E \mathrm{~cm}^{-1}$ \\
\hline 387.2 & 4 & 1 & 0.0 & 0.0 \\
5811.2 & 4 & 2 & -130.7 & 0.0 \\
9934.0 & 4 & 3 & 3.9 & -3.9 \\
10018.4 & 4 & 4 & 4.7 & 4.7 \\
10935.2 & 4 & 5 & 2.5 & -3.5 \\
11833.4 & 4 & 6 & 1.0 & 1.0 \\
12109.4 & 4 & 7 & 0.2 & -0.2 \\
17022.4 & 4 & 8 & 0.0 & 0.0 \\
\end{tabular}




\begin{tabular}{|c|c|c|c|c|}
\hline 18033.4 & 4 & 9 & 0.0 & 0.0 \\
\hline 18264.0 & 2 & 0 & 0.0 & -0.2 \\
\hline 18547.9 & 2 & 1 & 1.1 & 0.2 \\
\hline 19325.6 & 2 & 2 & 0.3 & 0.0 \\
\hline 19430.0 & 2 & 3 & 2.8 & 0.0 \\
\hline 20922.9 & 2 & 4 & 0.0 & 0.0 \\
\hline 23037.7 & 2 & 5 & 0.0 & 0.0 \\
\hline 23120.2 & 2 & 6 & 1.4 & 0.0 \\
\hline 23313.1 & 2 & 7 & -1.1 & 1.1 \\
\hline 23393.3 & 2 & 8 & -1.6 & -1.6 \\
\hline 23437.5 & 2 & 9 & 0.1 & 0.1 \\
\hline 23757.2 & 2 & 10 & 0.4 & 0.3 \\
\hline 26193.3 & 2 & 11 & -0.2 & 0.2 \\
\hline 27781.0 & 2 & 12 & 0.5 & 0.0 \\
\hline 27914.6 & 2 & 13 & 0.0 & 0.0 \\
\hline 28535.9 & 2 & 14 & -0.2 & 0.2 \\
\hline 29435.7 & 2 & 15 & 0.3 & 0.0 \\
\hline 29775.0 & 2 & 16 & 0.0 & 0.0 \\
\hline 30597.5 & 2 & 17 & 0.0 & 0.0 \\
\hline 31268.9 & 2 & 18 & 0.0 & 0.0 \\
\hline 31444.0 & 2 & 19 & 0.4 & 0.0 \\
\hline 32482.4 & 2 & 20 & -1.0 & -0.8 \\
\hline 32583.1 & 2 & 21 & -1.0 & 0.8 \\
\hline 33466.4 & 2 & 22 & -0.1 & 0.1 \\
\hline 35360.2 & 2 & 23 & 0.0 & 0.0 \\
\hline 38130.6 & 2 & 24 & 0.0 & 0.0 \\
\hline 38162.8 & 2 & 25 & 0.0 & 0.0 \\
\hline 38272.6 & 2 & 26 & 0.1 & 0.0 \\
\hline 38580.1 & 2 & 27 & 0.0 & 0.0 \\
\hline 40932.5 & 2 & 28 & 0.3 & 0.0 \\
\hline 41009.1 & 2 & 29 & 0.0 & 0.0 \\
\hline 42855.8 & 2 & 30 & 0.0 & 0.0 \\
\hline 42868.1 & 2 & 31 & 0.0 & 0.0 \\
\hline 42978.0 & 2 & 32 & 0.0 & 0.0 \\
\hline 44072.9 & 2 & 33 & 0.0 & 0.0 \\
\hline 46254.9 & 2 & 34 & 0.0 & 0.0 \\
\hline 61694.2 & 2 & 35 & 0.0 & 0.0 \\
\hline 63341.3 & 2 & 36 & 0.0 & 0.0 \\
\hline 63666.9 & 2 & 37 & 0.0 & 0.0 \\
\hline 65688.7 & 2 & 38 & 0.0 & 0.0 \\
\hline 65837.4 & 2 & 39 & 0.1 & 0.0 \\
\hline
\end{tabular}




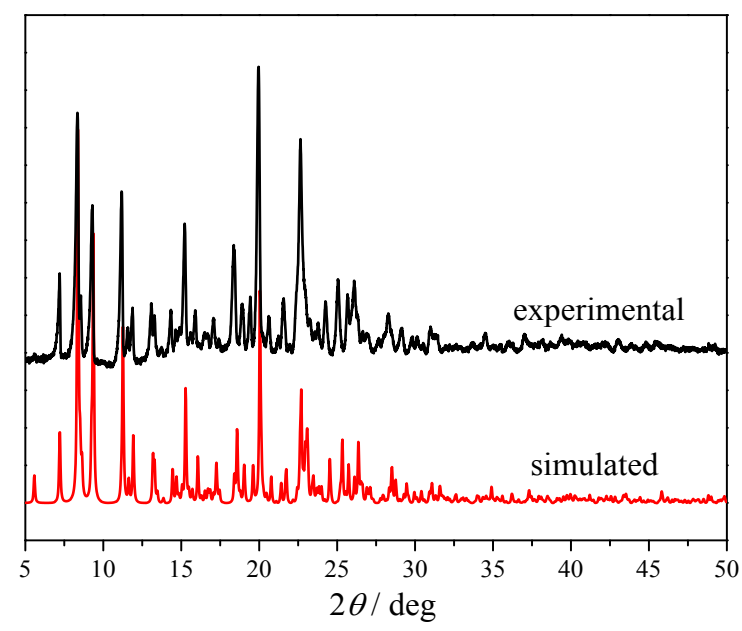

Figure S1. Powder X-ray diffraction data for $\mathbf{1}$. The red curves are calculated from the single crystal data.

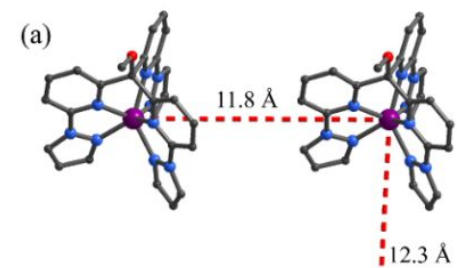

(b)
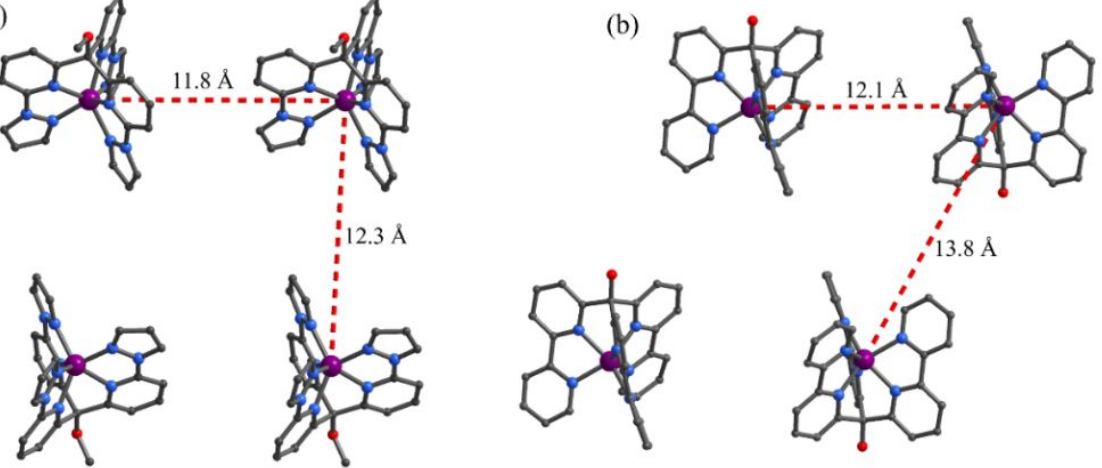

Figure S2. Packing diagrams of $\mathbf{1}$ (a) and $\mathbf{2}$ (b). The dashed lines indicate the nearest intermolecular Co $\cdots$ Co separations. Hydrogen atoms, counterions and interstitial solvents are omitted for the sake of clarify.

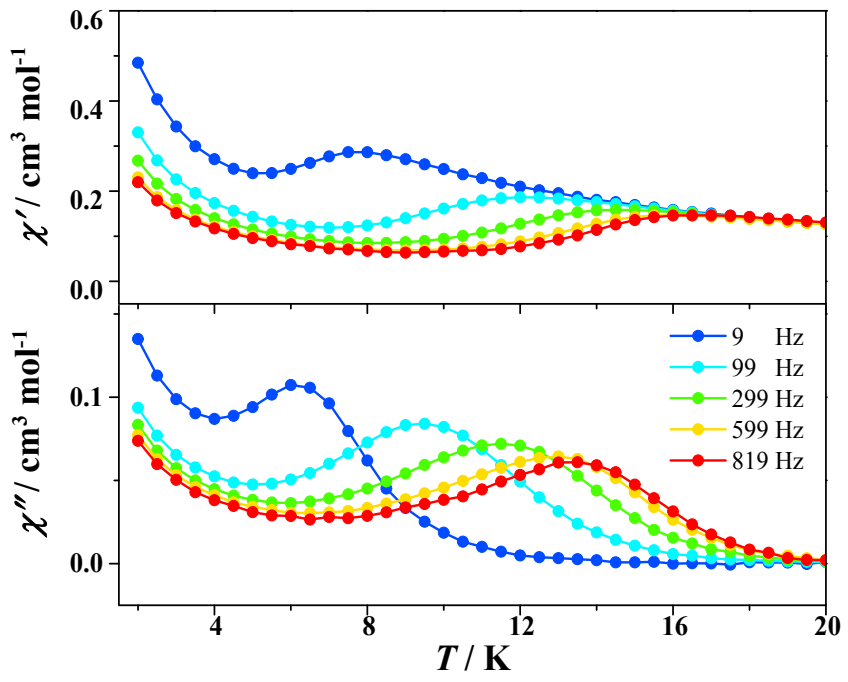

Figure S3. Temperature dependence of the in-phase $\left(\chi^{\prime}\right)$ and out-of-phase $\left(\chi^{\prime \prime}\right)$ ac susceptibility data for $\mathbf{1}$ under a zero applied dc field. The lines are guides to the eyes. 


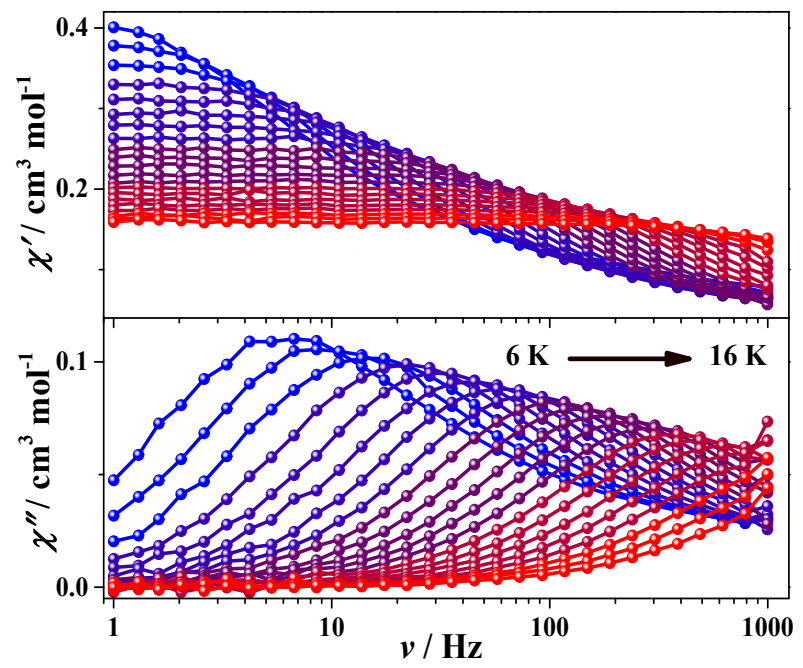

Figure S4. Frequency dependence of the in-phase $\left(\chi^{\prime}\right)$ and out-of-phase $\left(\chi^{\prime \prime}\right)$ ac susceptibility data for $\mathbf{1}$ under a zero applied dc field. The lines are guides to the eyes.

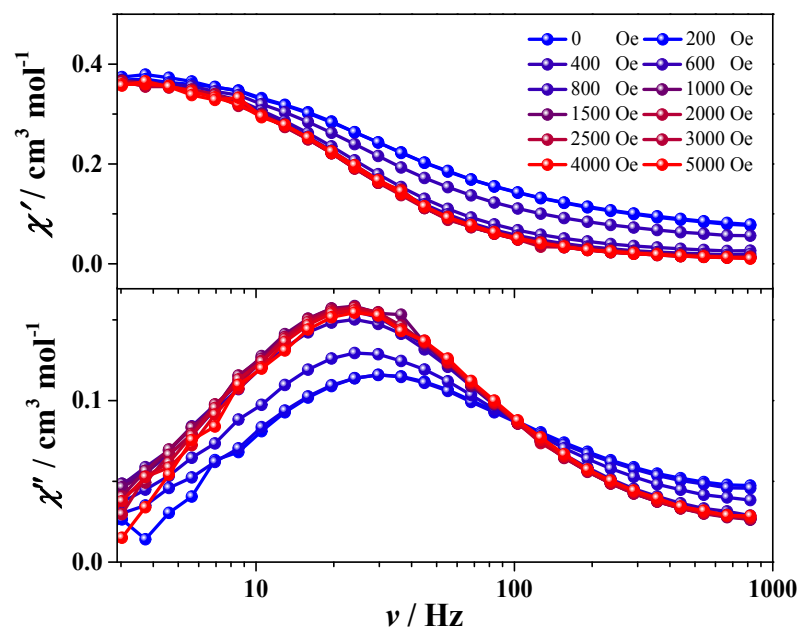

Figure S5. Frequency dependence of the in-phase $\left(\chi^{\prime}\right)$ and out-of-phase $\left(\chi^{\prime \prime}\right)$ ac susceptibility data for 1 as a function of applied field at $8 \mathrm{~K}$. The lines are guides to the eyes.

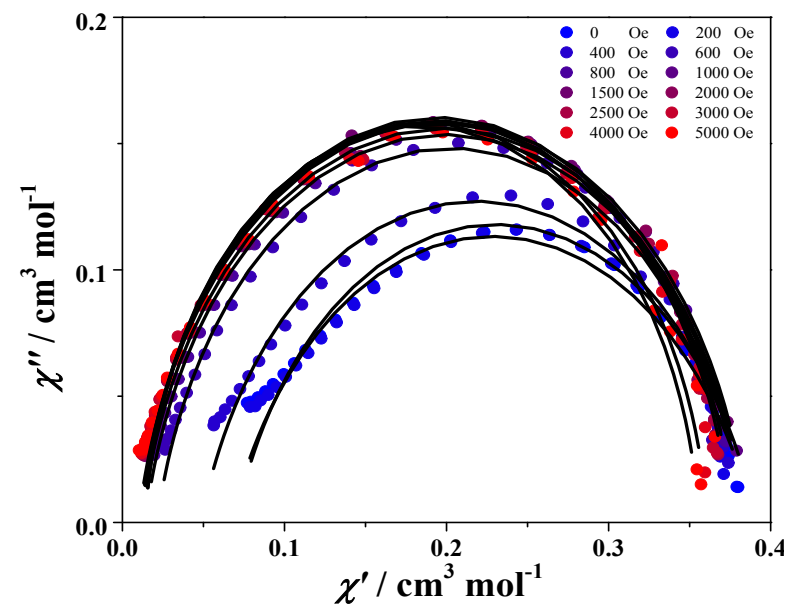

Figure S6. The Cole-Cole plots of 1 at $8 \mathrm{~K}$ and under different dc fields. The lines correspond to the fit to the data. 


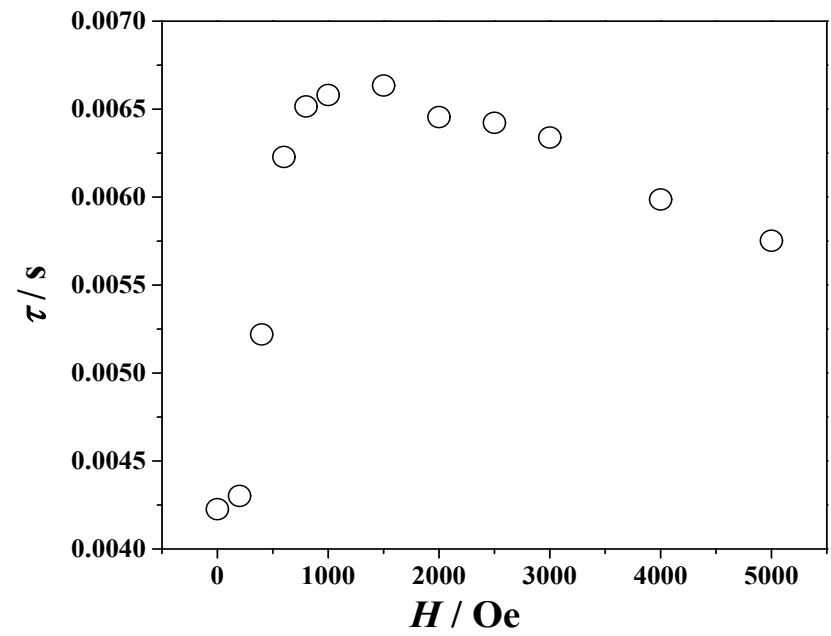

Figure S7. Field dependent relaxation times for 1 at $8 \mathrm{~K}$.

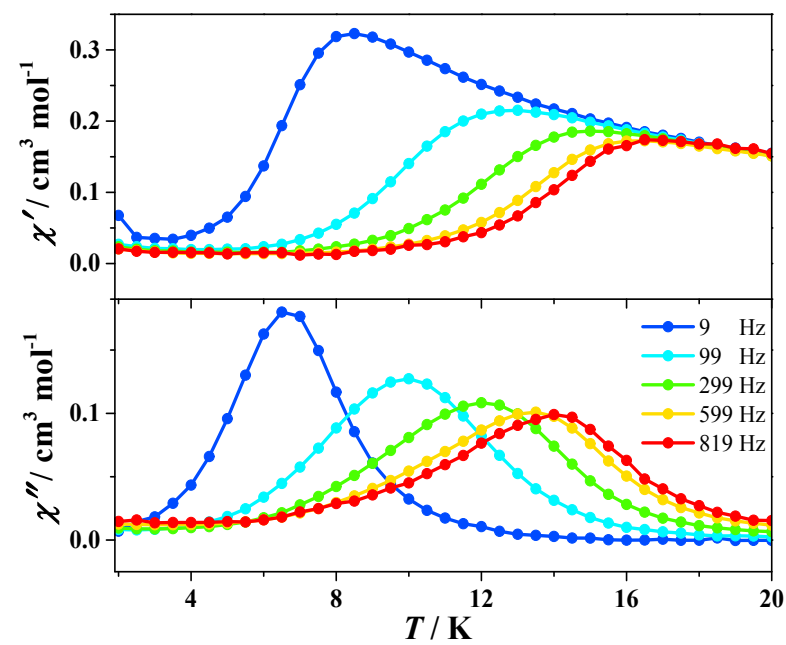

Figure S8. Temperature dependence of the in-phase $\left(\chi^{\prime}\right)$ and out-of-phase $\left(\chi^{\prime \prime}\right)$ ac susceptibility data for $\mathbf{1}$ under a 1000 Oe applied dc field. The lines are guides to the eyes.

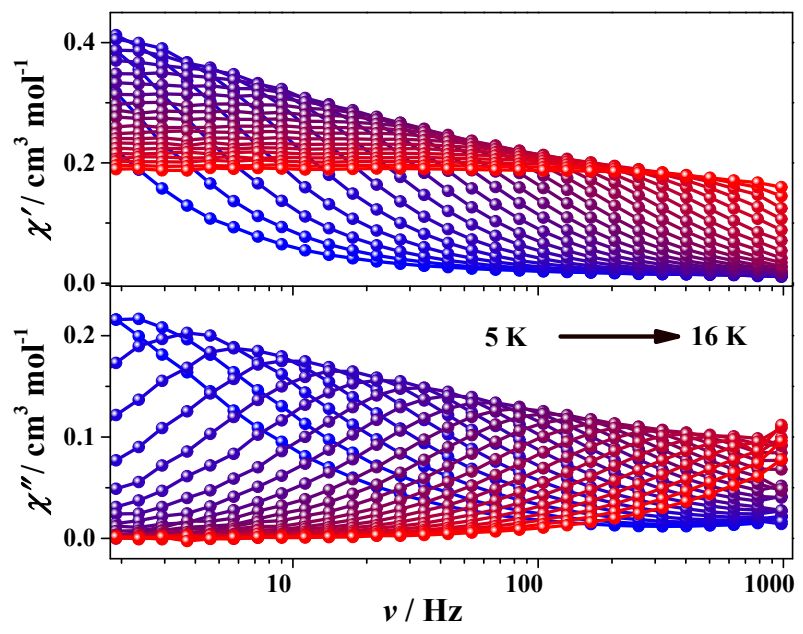

Figure S9. Frequency dependence of the in-phase $\left(\chi^{\prime}\right)$ and out-of-phase $\left(\chi^{\prime \prime}\right)$ ac susceptibility data for $\mathbf{1}$ under a 1000 Oe applied dc field. The lines are guides to the eyes. 


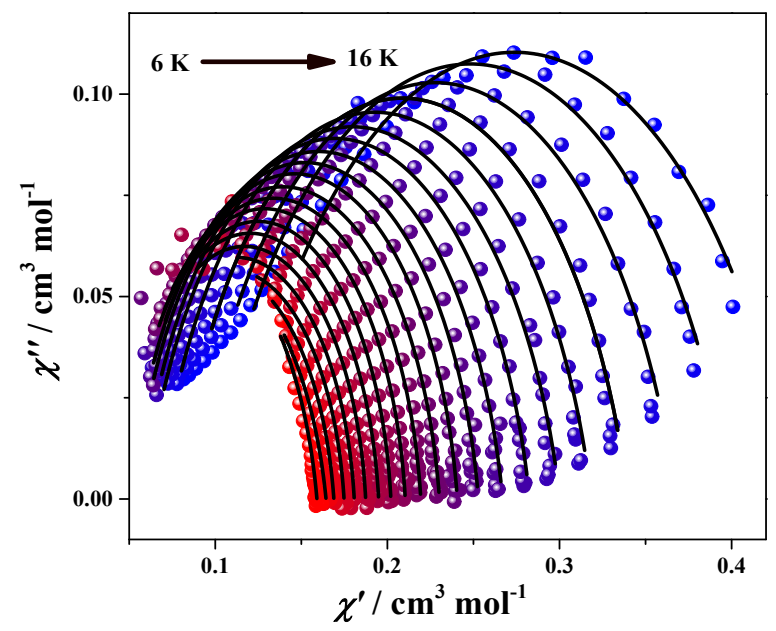

Figure S10. The Cole-Cole plots of 1 under a zero dc applied field. The lines represent the fit to the data.

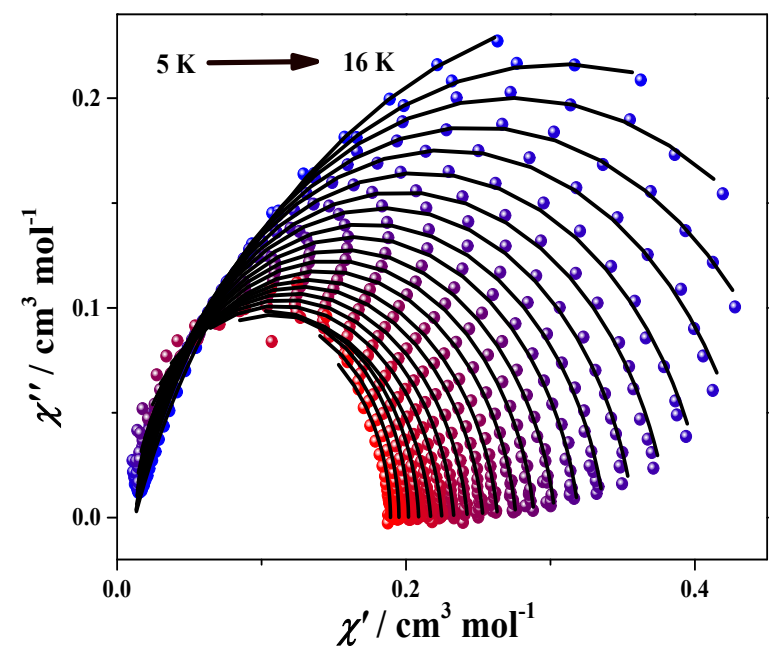

Figure S11. The Cole-Cole plots of 1 under a 1000 Oe dc appliedfield. The lines represent the fit to the data.

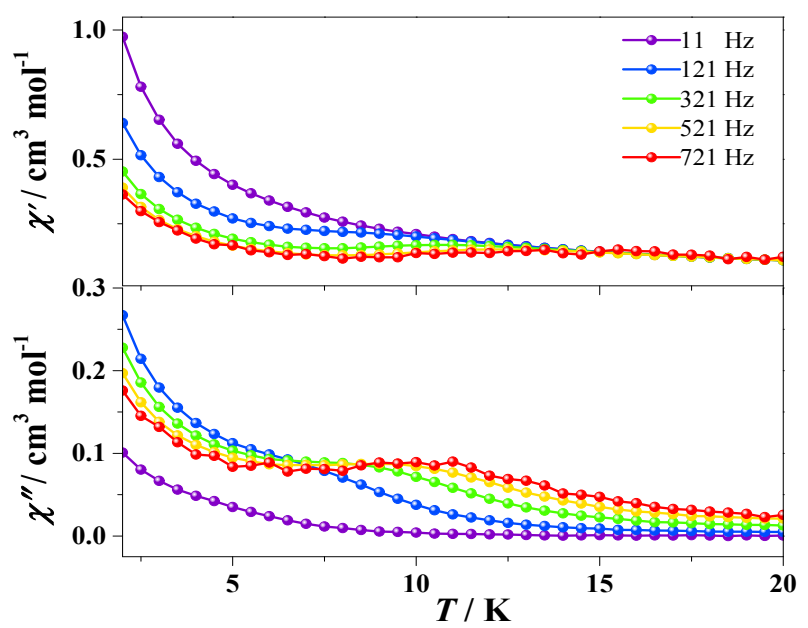

Figure S12. Temperature dependence of the in-phase $\left(\chi^{\prime}\right)$ and out-of-phase $\left(\chi^{\prime \prime}\right)$ ac susceptibility 
data for $\mathbf{2}$ under a zero dc applied field. The lines are guides to the eyes.

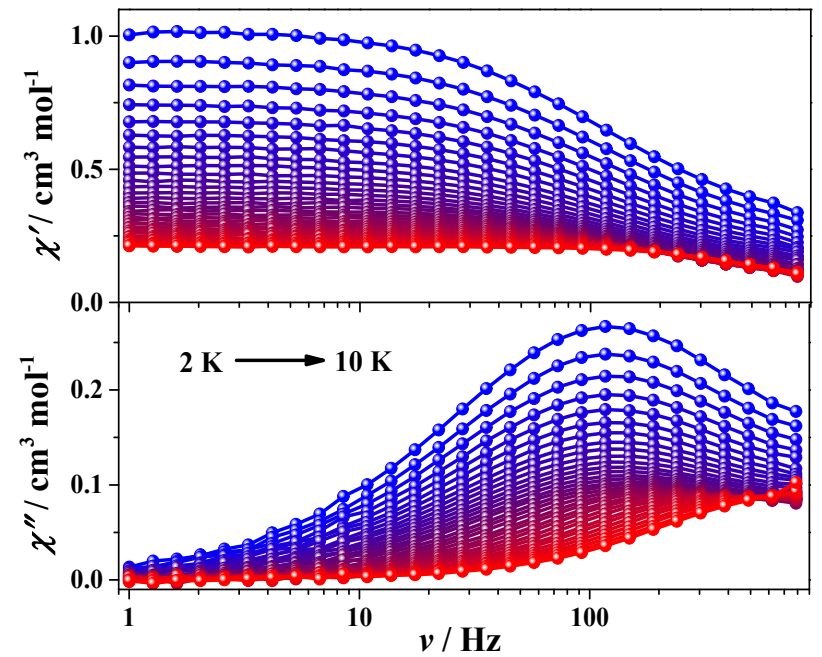

Figure S13. Frequency dependence of the in-phase $\left(\chi^{\prime}\right)$ and out-of-phase $\left(\chi^{\prime \prime}\right)$ ac susceptibility data for $\mathbf{2}$ under a zero dc applied field. The lines are guides to the eyes.

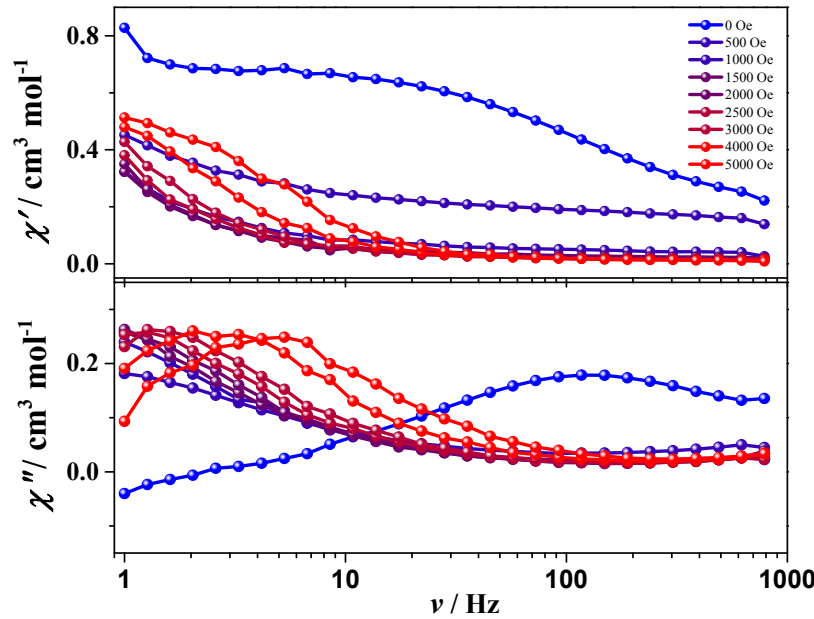

Figure S14. Frequency dependence of the in-phase $\left(\chi^{\prime}\right)$ and the out-of-phase $\left(\chi^{\prime \prime}\right)$ ac susceptibility data for $\mathbf{2}$ as a function of applied field at $3 \mathrm{~K}$. The lines are guides to the eyes.

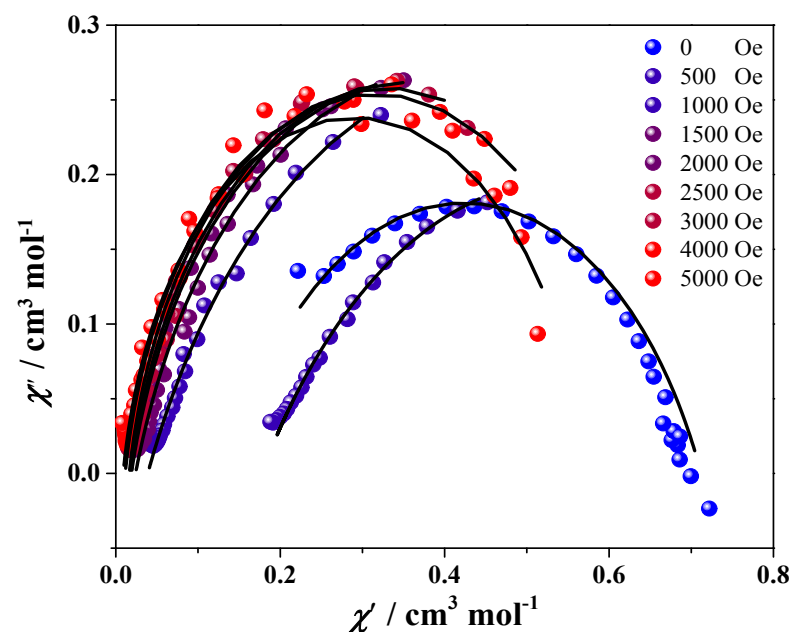

Figure S15. The Cole-Cole plots of 2 at $3 \mathrm{~K}$ and under different dc fields. The lines correspond to 
the fit to the data.s

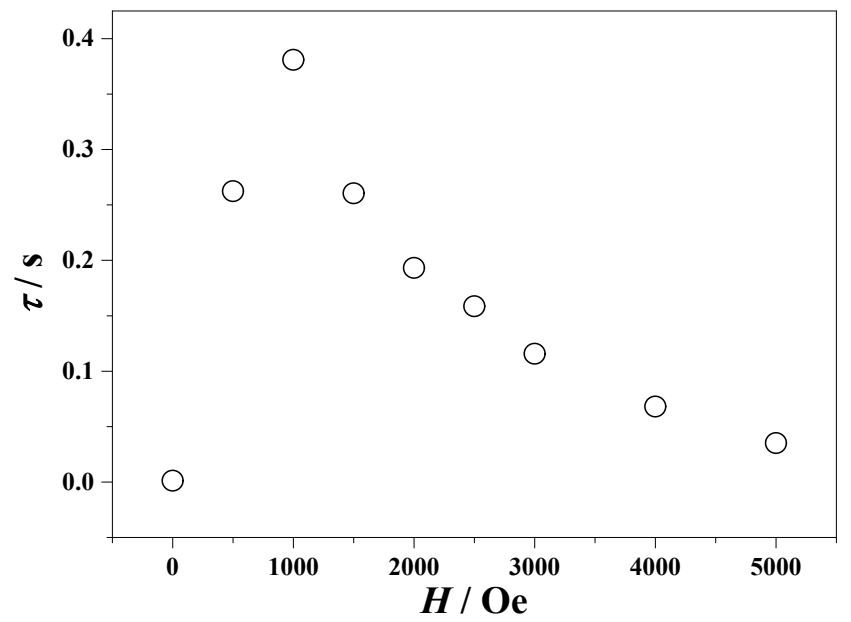

Figure S16. Field dependent relaxation times for 2 at $3 \mathrm{~K}$.

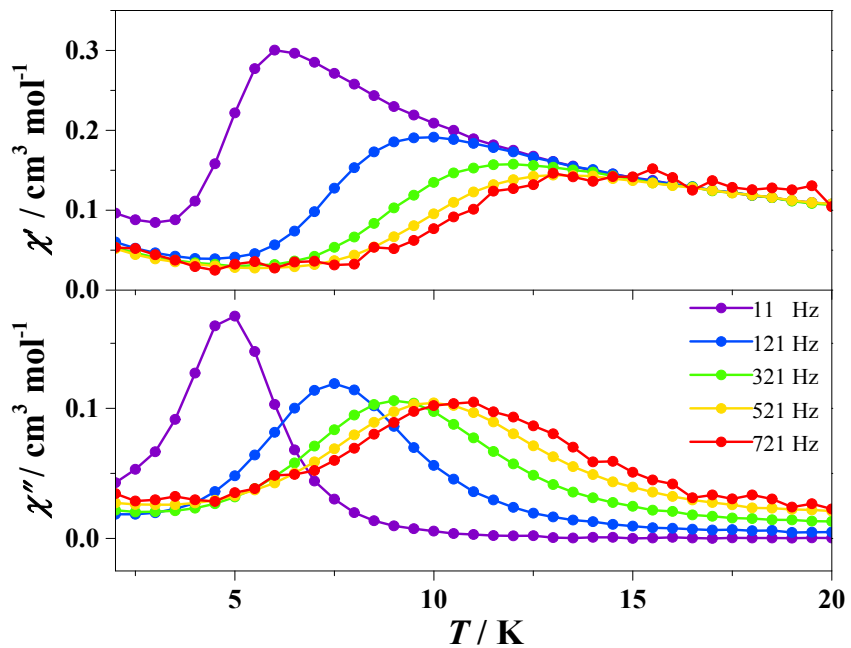

Figure S17. Temperature dependence of the in-phase $\left(\chi^{\prime}\right)$ and out-of-phase $\left(\chi^{\prime \prime}\right)$ ac susceptibility data for 2 under a 1000 Oe dc applied field. The lines are guides to the eyes.

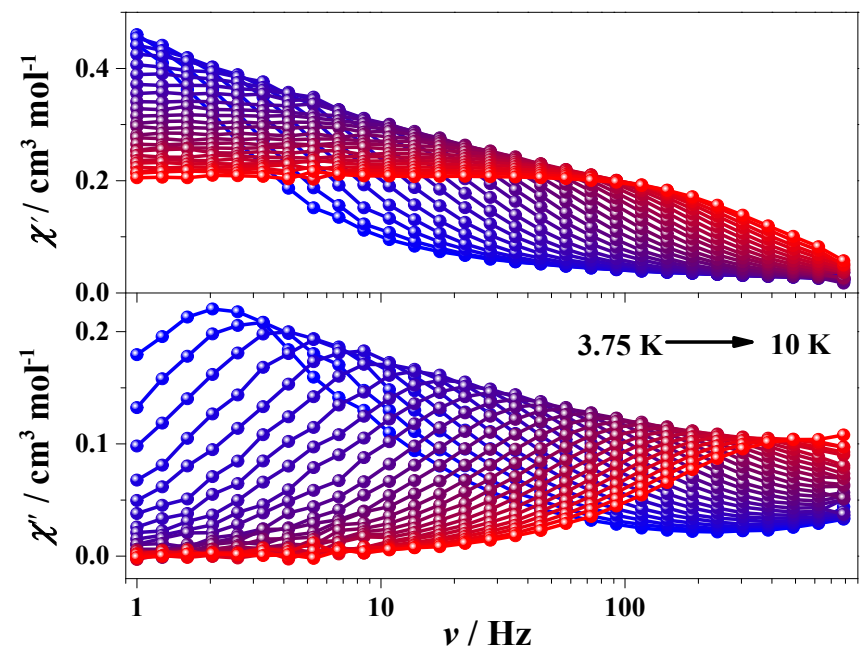

Figure S18. Frequency dependence of the in-phase $\left(\chi^{\prime}\right)$ and out-of-phase $\left(\chi^{\prime \prime}\right)$ ac susceptibility data 
for $\mathbf{2}$ under a1000 Oe dc applied field. The lines are guides to the eyes.

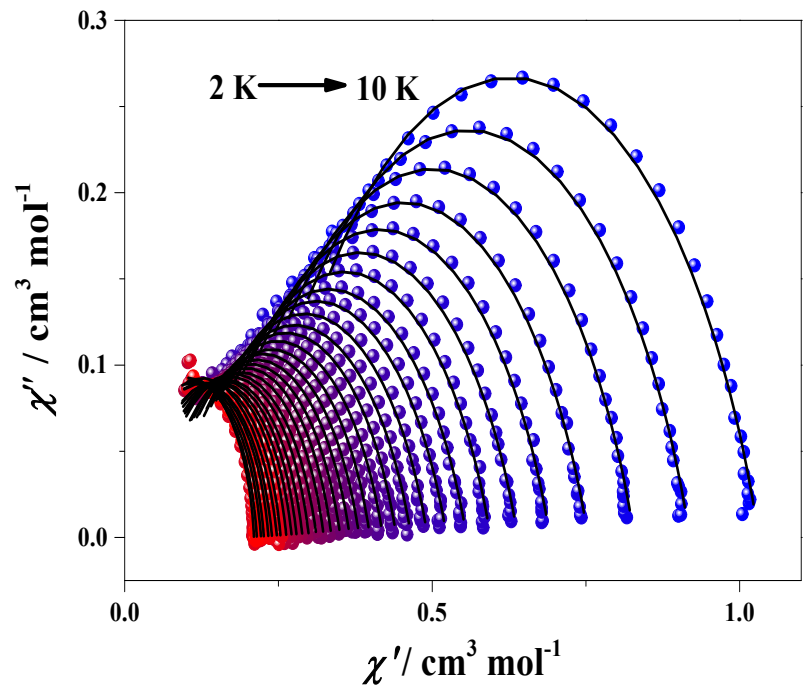

Figure S19. Cole-Cole plots of 2 under a zero dc field. The lines represent the fit to the data.

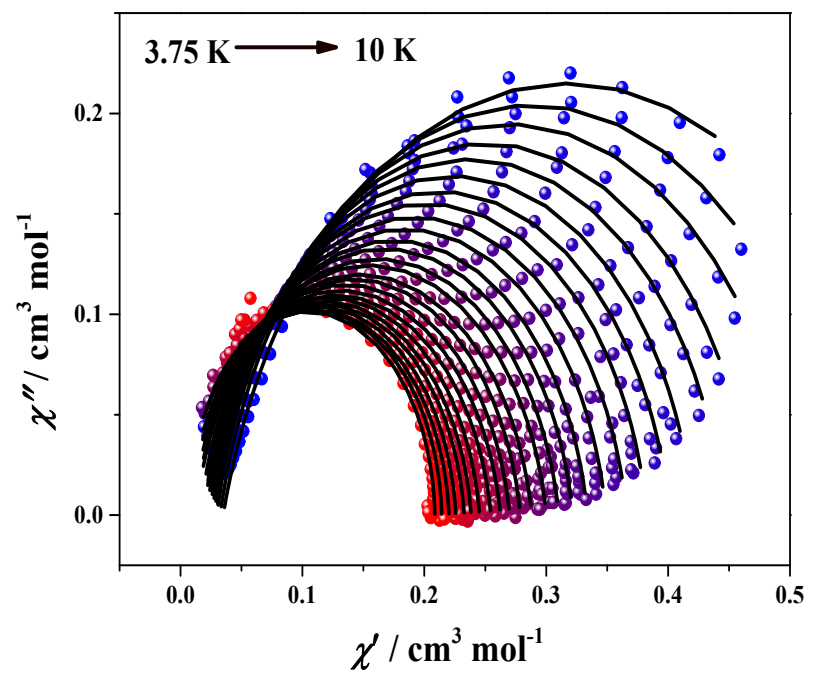

Figure S20. Cole-Cole plots of 2 under a 1000 Oe dc applied field. The lines correspond to a fit to the data.

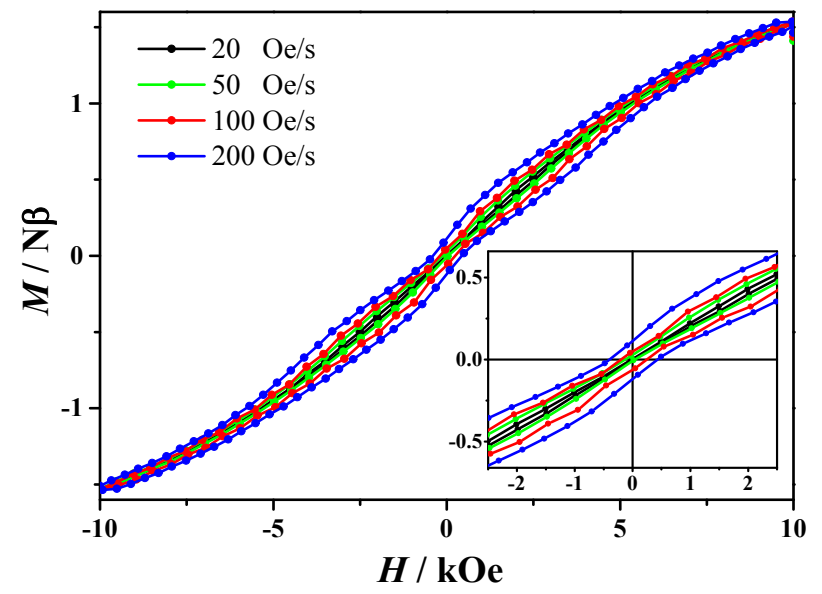

Figure S21. The magnetic hysteresis loops for 1 recorded at $2.0 \mathrm{~K}$ with different sweep rates. 


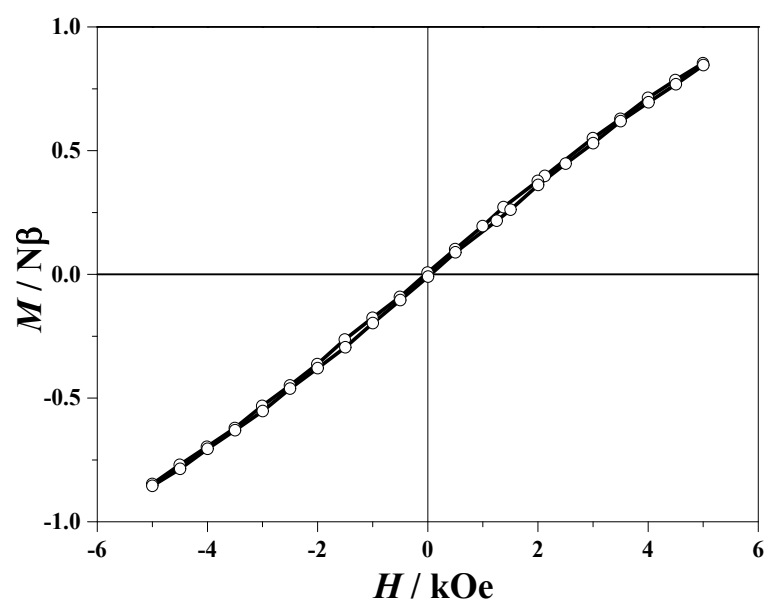

Figure S22. The magnetic hysteresis loops for 2 recorded at $2 \mathrm{~K}$ with a sweep rate of 30 Oe/s.
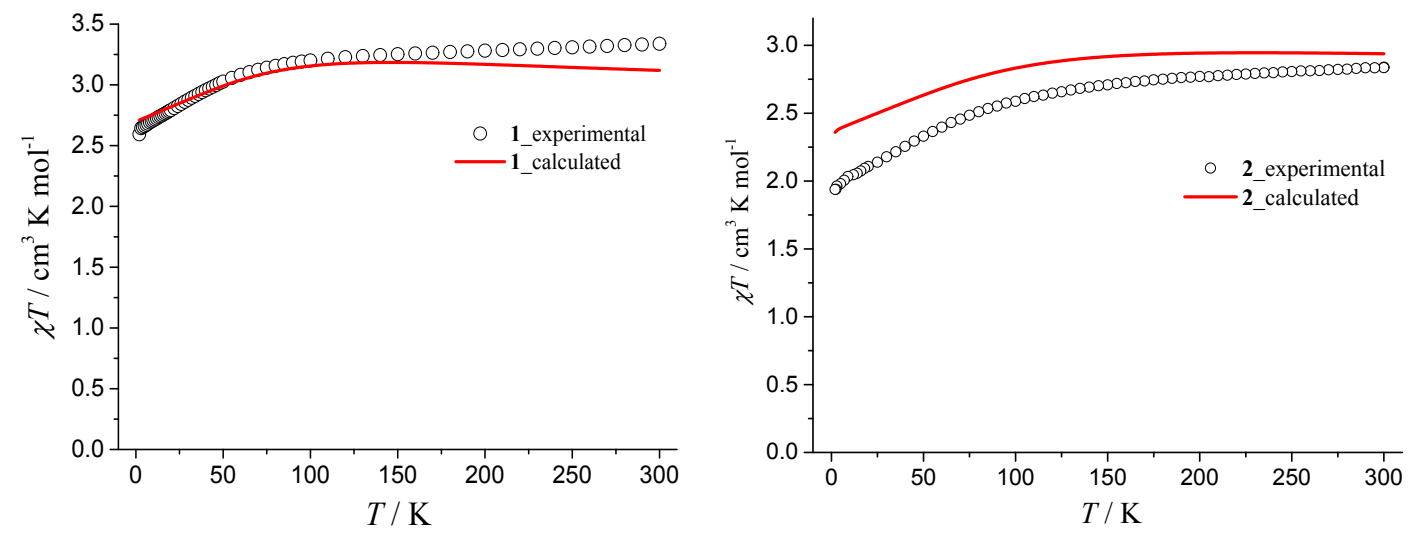

Figure S23. The dc susceptibilities of 1 and $\mathbf{2}$ reproduced with the NEVPT2-calculated spin Hamiltonian parameters.
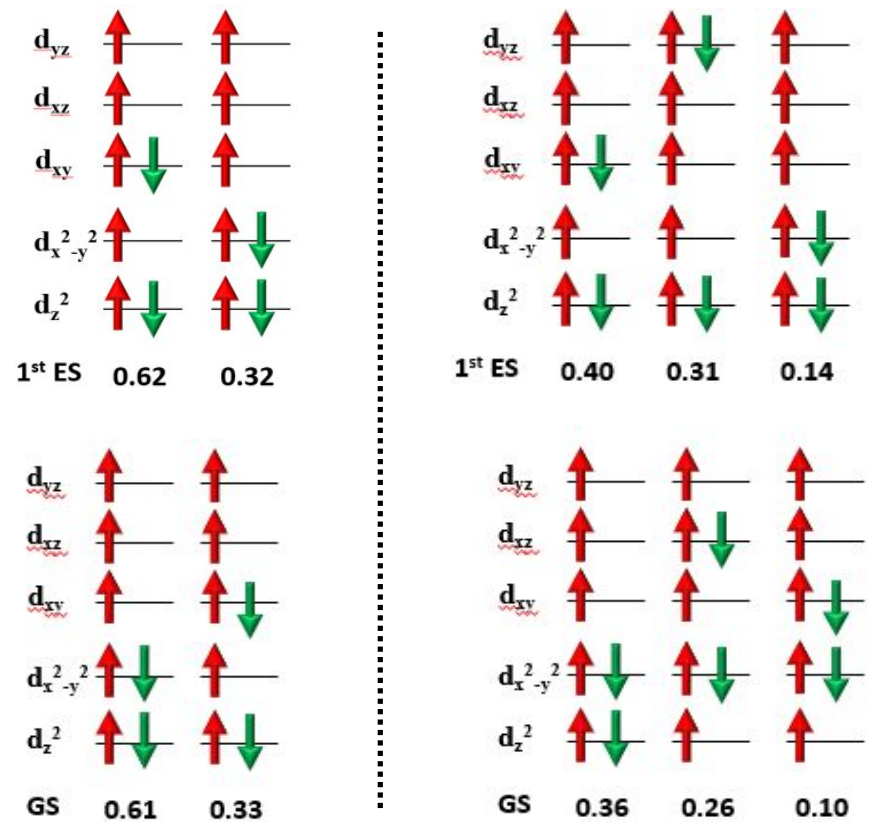

Figure S24. The multi-determinant characteristics of the ground and first excited state wave function for (a) 1 and (b) 2. Only computed CI coefficients that are larger than $10 \%$ are shown 
above.
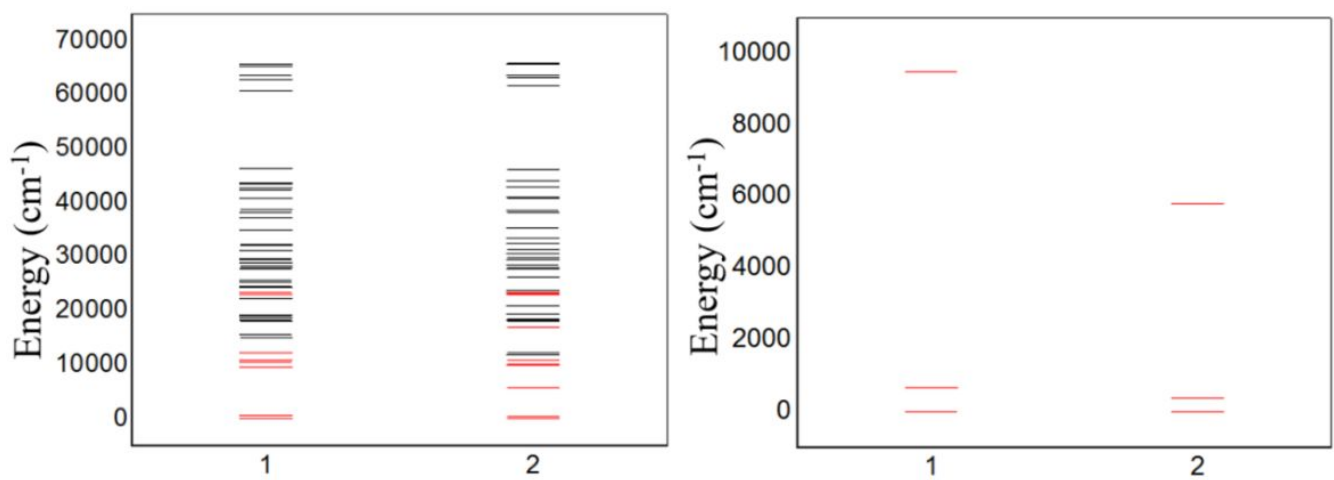

Figure S25. Ab initio NEVPT2+SA-CASSCF computed energies (left) for all roots in 1-2 and (right) energies of lower three roots in 1-2. Black = quartet energy levels and red = doublet energy levels. Doublets are higher in energy suggesting less aid to anisotropy parameters from spin flip transitions.

\section{Reference}

(1) (a) Li, X.; Gibb, C. L. D.; Kuebel, M. E.; Gibb, B. C., Two new ligands for carbonic anhydrase mimicry. Tetrahedron 2001, 57, 1175-1182. (b) Knight, J. C.; Amoroso, A. J.; Edwards, P. G.; Singh, N.; Ward, B. D., Shaping and enforcing coordination spheres: probing the ability of tripodal ligands to favour trigonal prismatic geometry. Dalton Trans. 2016, 45, 10630-10642. (c) Knight, J. C.; Alvarez, S.; Amoroso, A. J.; Edwards, P. G.; Singh, N., A novel bipyridine-based hexadentate tripodal framework with a strong preference for trigonal prismatic co-ordination geometries. Dalton Trans. 2010, 39, 3870-3883. (d) Yao, B.; Deng, Y.-F.; Li, T.; Xiong, J.; Wang, B.-W.; Zheng, Z.; Zhang, Y.-Z., Construction and Magnetic Study of a Trigonal-Prismatic Cobalt(II) Single-Ion Magnet. Inorg. Chem. 2018, 57, 14047-14051.

(2) (a) Carlin, R. L., Magnetochemistry. Springer-Verlag Press: Berlin, Heidelbeg, 1986. (b) Bain, G. A.; Berry, J. F., Diamagnetic Corrections and Pascal's Constants. J. Chem. Educ. 2008, 85, 532.

(3) (a) Sheldrick, G. M., SADABS, v.2.01, Bruker/Siemens Area Detector Absorption Correction Program. Bruker AXS: Madison, Wisconism, 1998. (b) Sheldrick, G. M., SHELXL-2014, Program for Crystal Structure Refinement. University of Göttingen, Göttingen, Germany, 2014. (c) Sheldrick, G. M., SHELXL-2014, Program for the solution of crystal structures. University of Göttingen, Göttingen, Germany, 2014; Vol. 8.

(4) Neese, F., Software update: the ORCA program system, version 4.0. WIREs Comput. Mol. Sci. 2018, 8, e1327.

(5) Aquilante, F.; Autschbach, J.; Carlson, R. K.; Chibotaru, L. F.; Delcey, M. G.; De Vico, L.; Fdez Galván, I.; Ferré, N.; Frutos, L. M.; Gagliardi, L.; Garavelli, M.; Giussani, A.; Hoyer, C. E.; Li Manni, G.; Lischka, H.; Ma, D.; Malmqvist, P. Å.; Müller, T.; Nenov, A.; Olivucci, M.; Pedersen, T. B.; Peng, D.; Plasser, F.; Pritchard, B.; Reiher, M.; Rivalta, I.; Schapiro, I.; Segarra-Martí, J.; Stenrup, M.; Truhlar, D. G.; Ungur, L.; Valentini, A.; Vancoillie, S.; Veryazov, V.; Vysotskiy, V. P.; Weingart, O.; Zapata, F.; Lindh, R., Molcas 8: New capabilities for multiconfigurational quantum chemical calculations across the periodic table. J. Comput. Chem. 2016, 37, 506-541. (6) Herchel, R.; Vahovska, L.; Potocnak, I.; Travnicek, Z., Slow magnetic relaxation in octahedral cobalt(II) fieldinduced single-ion magnet with positive axial and large rhombic anisotropy. Inorg. Chem. 2014, 53, 5896-5898.

(7) van Wüllen, C., Molecular density functional calculations in the regular relativistic approximation: Method, application to coinage metal diatomics, hydrides, fluorides and chlorides, and comparison with first-order relativistic calculations. J. Chem. Phys. 1998, 109, 392-399.

(8) Weigend, F.; Ahlrichs, R., Balanced basis sets of split valence, triple zeta valence and quadruple zeta valence quality for H to Rn: Design and assessment of accuracy. Phys. Chem. Chem. Phys. 2005, 7, 3297-3305. 
(9) (a) Angeli, C.; Cimiraglia, R.; Evangelisti, S.; Leininger, T.; Malrieu, J. P., Introduction of n-electron valence states for multireference perturbation theory. J. Chem. Phys. 2001, 114, 10252. (b) Angeli, C.; Cimiraglia, R.; Malrieu, J. P., N-electron valence state perturbation theory: a fast implementation of the strongly contracted variant. Chem. Phys. Lett. 2001, 350, 297-305. (c) Angeli, C.; Cimiraglia, R.; Malrieu, J. P., n-electron valence state perturbation theory: A spinless formulation and an efficient implementation of the strongly contracted and of the partially contracted variants. J. Chem. Phys. 2002, 117, 9138. (d) Angeli, C.; Borini, S.; Cestari, M.; Cimiraglia, R., A quasidegenerate formulation of the second order n-electron valence state perturbation theory approach. J. Chem. Phys. 2004, 121, 4043.

(10) (a) Cremades, E.; Ruiz, E., Mononuclear FeII Single-Molecule Magnets: A Theoretical Approach. Inorg. Chem. 2011, 50, 4016-4020. (b) Maurice, R.; Vendier, L.; Costes, J.-P., Magnetic Anisotropy in NiII-YIII Binuclear Complexes: On the Importance of Both the First Coordination Sphere of the NiII Ion and the YIII Ion Belonging to the Second Coordination Sphere. Inorg. Chem. 2011, 50, 11075-11081. (c) Maganas, D.; Sottini, S.; Kyritsis, P.; Groenen, E. J.; Neese, F., Theoretical analysis of the spin Hamiltonian parameters in Co(II)S4 complexes, using density functional theory and correlated ab initio methods. Inorg. Chem. 2011, 50, 8741-8754. (d) Ganyushin, D.; Neese, F., First-principles calculations of zero-field splitting parameters. J. Chem. Phys. 2006, 125, 024103. (e) Neese, F., Importance of Direct Spin-Spin Coupling and Spin-Flip Excitations for the Zero-Field Splittings of Transition Metal Complexes: A Case Study. J. Am. Chem. Soc. 2006, 128, 10213-10222. (f) Neese, F.; Solomon, E. I., Interpretation and Calculation of Spin-Hamiltonian Parameters in Transition Metal Complexes. Magnetism: Molecules to Materials 2004. (g) Neese, F.; Solomon, E. I., Calculation of Zero-Field Splittings, g-Values, and the Relativistic Nephelauxetic Effect in Transition Metal Complexes. Application to High-Spin Ferric Complexes. Inorg. Chem. 1998, 37, 6568-6582.

(11) (a) Chibotaru, L. F.; Ungur, L., Ab initio calculation of anisotropic magnetic properties of complexes. I. Unique definition of pseudospin Hamiltonians and their derivation. J. Chem. Phys. 2012, 137, 064112. (b) Granovsky, A. A., Extended multi-configuration quasi-degenerate perturbation theory: The new approach to multi-state multireference perturbation theory. J. Chem. Phys. 2011, 134, 214113.

(12) Ungur, L.; Thewissen, M.; Costes, J. P.; Wernsdorfer, W.; Chibotaru, L. F., Interplay of Strongly Anisotropic Metal Ions in Magnetic Blocking of Complexes. Inorg. Chem. 2013, 52, 6328-6337.

(13) (a) Guo, Y.-N.; Xu, G.-F.; Guo, Y.; Tang, J., Relaxation dynamics of dysprosium(iii) single molecule magnets. Dalton. Trans., 2011, 40, 9953-9963; (b) Reta, D.; Chilton, N. F., Uncertainty estimates for magnetic relaxation times and magnetic relaxation parameters. Phys. Chem. Chem. Phys. 2019, 21, 23567-23575.

(14) Zhu, Y.-Y.; Cui, C.; Zhang, Y.-Q.; Jia, J.-H.; Guo, X.; Gao, C.; Qian, K.; Jiang, S.-D.; Wang, B.-W.; Wang, Z.M.; Gao, S., Zero-field slow magnetic relaxation from single Co(ii) ion: a transition metal single-molecule magnet with high anisotropy barrier. Chem. Sci. 2013, 4, 1802-1806.

(15) Gomez-Coca, S.; Cremades, E.; Aliaga-Alcalde, N.; Ruiz, E., Mononuclear single-molecule magnets: tailoring the magnetic anisotropy of first-row transition-metal complexes. J. Am. Chem. Soc. 2013, 135, 7010-7018.

(16) Novikov, V. V.; Pavlov, A. A.; Nelyubina, Y. V.; Boulon, M.-E.; Varzatskii, O. A.; Voloshin, Y. Z.; Winpenny, R. E. P., A Trigonal Prismatic Mononuclear Cobalt(II) Complex Showing Single-Molecule Magnet Behavior. J. Am. Chem. Soc. 2015, 137, 9792-9795.

(17) Zhu, Y.-Y.; Zhang, Y.-Q.; Yin, T.-T.; Gao, C.; Wang, B.-W.; Gao, S., A Family of $\mathrm{Co}^{\mathrm{III}} \mathrm{Co}_{3}^{\mathrm{III}}{ }_{3}$ Single-Ion Magnets with Zero-Field Slow Magnetic Relaxation: Fine Tuning of Energy Barrier by Remote Substituent and Counter Cation. Inorg. Chem. 2015, 54, 5475-5486.

(18) Villa-Pérez, C.; Oyarzabal, I.; Echeverría, G. A.; Valencia-Uribe, G. C.; Seco, J. M.; Soria, D. B., Single-Ion Magnets Based on Mononuclear Cobalt(II) Complexes with Sulfadiazine. Eur. J. Inorg. Chem. 2016, 4835-4841.

(19) Peng, Y.; Bodenstein, T.; Fink, K.; Mereacre, V.; Anson, C. E.; Powell, A. K., Magnetic anisotropy of a Co ${ }^{\text {II }}$ 
single ion magnet with distorted trigonal prismatic coordination: theory and experiment. Phys. Chem. Chem. Phys. 2016, 18, 30135-30143.

(20) Pavlov, A. A.; Nelyubina, Y. V.; Kats, S. V.; Penkova, L. V.; Efimov, N. N.; Dmitrienko, A. O.; Vologzhanina, A. V.; Belov, A. S.; Voloshin, Y. Z.; Novikov, V. V., Polymorphism in a Cobalt-Based Single-Ion Magnet Tuning Its Barrier to Magnetization Relaxation. J. Phys. Chem. Lett. 2016, 7, 4111-4116.

(21) Ozumerzifon, T. J.; Bhowmick, I.; Spaller, W. C.; Rappé, A. K.; Shores, M. P., Toward steric control of guest binding modality: a cationic Co(ii) complex exhibiting cation binding and zero-field relaxation. Chem. Commun. 2017, 53, 4211-4214.

(22) Pavlov, A. A.; Savkina, S. A.; Belov, A. S.; Nelyubina, Y. V.; Efimov, N. N.; Voloshin, Y. Z.; Novikov, V. V., Trigonal Prismatic Tris-pyridineoximate Transition Metal Complexes: A Cobalt(II) Compound with High Magnetic Anisotropy. Inorg. Chem. 2017, 56, 6943-6951. 